\title{
Wild-type and splice-variant secretin receptors in lung cancer: overexpression in carcinoid tumors and peritumoral lung tissue
}

\author{
Meike U Körner ${ }^{1}$, Gregory M Hayes ${ }^{2}$, Patricia E Carrigan ${ }^{2}$, Ruth Rehmann ${ }^{1}$, \\ Laurence J Miller ${ }^{2}$ and Jean C Reubi ${ }^{1}$ \\ ${ }^{1}$ Division of Cell Biology and Experimental Cancer Research, Institute of Pathology of the University of Bern, \\ Bern, Switzerland and ${ }^{2}$ Cancer Center, Mayo Clinic, Scottsdale, AZ, USA
}

\begin{abstract}
Gastrointestinal peptide hormone receptors, like somatostatin receptors, are often overexpressed in human cancer, allowing receptor-targeted tumor imaging and therapy. A novel candidate for these applications is the secretin receptor recently identified in pancreatic and cholangiocellular carcinomas. In the present study, secretin receptors were assessed in a non-gastrointestinal tissue, the human lung. Non-small-cell lung cancers $(n=26)$, small-cell lung cancers $(n=10)$, bronchopulmonary carcinoid tumors $(n=29)$, and non-neoplastic lung $(n=46)$ were investigated for secretin receptor protein expression with in vitro receptor autoradiography, using ${ }^{125} \mathrm{I}-\left[\mathrm{Tyr}^{10}\right]$ rat secretin and for secretin receptor transcripts with RT-PCR. Secretin receptor protein expression was found in $62 \%$ of bronchopulmonary carcinoids in moderate to high density, in $12 \%$ of non-small cell lung cancers in low density, but not in small cell lung cancers. In tumors found to be secretin receptor positive by autoradiography, RT-PCR revealed transcripts for the wild-type secretin receptor and for novel secretin receptor splice variants. In the non-neoplastic lung, secretin receptor protein expression was observed in low density along the alveolar septa in direct tumor vicinity in cases of acute inflammation, but not in histologically normal lung. In the autoradiographically positive peritumoral lung, RT-PCR showed transcripts for the wild-type secretin receptor and for a secretin receptor spliceoform different from those occurring in lung and gut tumors. In conclusion, secretin receptors are new markers for bronchopulmonary carcinoid tumors, and represent the molecular basis for an in vivo targeting of carcinoid tumors for diagnosis and therapy. Furthermore, secretin receptors may play a role in peritumoral lung pathophysiology. Secretin receptor mis-splicing specifically occurs in tumor and non-tumor lung pathology.
\end{abstract}

Modern Pathology (2008) 21, 387-395; doi:10.1038/modpathol.3801005; published online 25 January 2008

Keywords: secretin receptor; carcinoid tumor; lung; splice variant; receptor autoradiography; RT-PCR

Many gastrointestinal peptide hormone receptors are overexpressed in human cancer. This receptor overexpression represents the molecular basis for an in vivo targeting of tumors with radiolabeled peptide analogs for diagnostic and therapeutic purposes. ${ }^{1}$ Somatostatin receptor targeting is the clinically best established example: gastroenteropancreatic neuroendocrine tumors with high somatostatin receptor overexpression are imaged scintigraphically with radiolabeled somatostatin analogs like OctreoScan ${ }^{\circledR}$, with high sensitivity; ${ }^{2,3}$ moreover, these tumors are

Correspondence: Professor JC Reubi, MD, Division of Cell Biology and Experimental Cancer Research, Institute of Pathology of the University of Bern, Murtenstrasse 31, PO Box 62, Bern CH-3010, Switzerland.

E-mail: reubi@pathology.unibe.ch

Received 23 July 2007; revised 03 October 2007; accepted 26 November 2007; published online 25 January 2008 highly responsive to targeted therapy with radiotoxic somatostatin analogs. ${ }^{4,5}$ The good results of somatostatin receptor targeting have stimulated the search for other peptide hormone receptors suitable for an in vivo targeting of tumors, in order to be able to target a wider spectrum of tumors. The secretin receptor is a novel promising candidate for this purpose.

The secretin receptor is a $G$ protein-coupled receptor. Together with VIP, glucagon, and other receptors it forms the secretin receptor family. ${ }^{6}$ The secretin receptor has long been known for its important digestive functions in the pancreas and biliary tract. ${ }^{7}$ Only recently was it recognized that the secretin receptor also plays a role in pathology, namely in cancer arising from physiological secretin targets. Pancreatic ductal adenocarcinomas, cholangiocellular carcinomas, and gastrinomas were found to show high secretin receptor expression. 
Moreover, these tumors expressed not only the wild-type secretin receptor, but also transcripts for various secretin receptor splice variants arising from alternative splicing of the receptor pre-mRNA. ${ }^{8-12}$ The splice-variant receptors may exert functional effects on the tumor cells, which remain incompletely characterized. For example, the secretin receptor splice variant with exon 3 deletion silences the wild-type receptor and thus may inhibit wildtype receptor-mediated functions such as hormone secretion or proliferation..$^{8,9,13}$ Furthermore, the secretin receptor spliceoform with exon 3 and 4 deletion might be secreted by pancreatic ductal adenocarcinomas and can be measured in the patients' blood. ${ }^{12}$ Thus, the secretin receptor represents a potential new target for diagnosis and therapy of human cancer.

While the secretin receptor is extensively investigated in the gastrointestinal tract, only little is known about its expression and functions in other organs. Concerning the human lung, single studies reported secretin receptor mRNA in lung membrane preparations ${ }^{14,15}$ and functional secretin receptor protein in the bronchus epithelium. ${ }^{16}$ Since in the gastrointestinal tract secretin receptors are found in tumors arising from tissues with physiological secretin receptor expression, it appeared promising to investigate human lung cancer for secretin receptors. Therefore, in the present study, various lung tumors, as well as normal lung, were assessed for secretin receptor protein by in vitro secretin receptor autoradiography, and for secretin receptor transcripts by RT-PCR.

\section{Materials and methods}

\section{Tissues}

The following human tissue samples obtained from surgical tumor resection specimens were included in the study: 29 bronchopulmonary carcinoid tumors ( 22 typical, 7 atypical ${ }^{17,18}$ ), 26 non-small cell lung cancers (NSCLC; 15 squamous cell carcinomas, 11 adenocarcinomas), 10 small cell lung cancers (SCLC), 11 mesotheliomas (10 pleural, 1 peritoneal), and 16 normal lung samples distant from tumors. In 32 specimens, peritumoral non-neoplastic lung was also present for investigation. The tissues were snap frozen and stored at $-80^{\circ} \mathrm{C}$.

The study conformed to the ethical guidelines of the Institute of Pathology of the University of Bern and was reviewed by the Institutional Review Board.

\section{In Vitro Secretin Receptor Autoradiography}

All cases were investigated for secretin receptor protein on the basis of specific binding of radioiodinated secretin using in vitro secretin receptor autoradiography. The procedure was carried out as described before. ${ }^{10}$ Cryostat sections (20- $\mu \mathrm{m}$ thick) mounted on glass slides were preincubated in $0.01 \mathrm{M}$ HEPES buffer ( $\mathrm{pH}$ 7.4) for $5 \mathrm{~min}$ at room temperature. Then they were incubated for $120 \mathrm{~min}$ at room temperature in the incubation solution containing HEPES buffer, $1 \%$ BSA, $130 \mathrm{mM} \mathrm{NaCl}$, $4.7 \mathrm{mM} \mathrm{KCl}, 5 \mathrm{mM}$ (Manganese(II)-chloride) $\cdot 4 \mathrm{H}_{2} \mathrm{O}$, $1 \mathrm{mM}$ EGTA, $1 \mathrm{mg} / \mathrm{ml}$ bacitracin, and 24000 c.p.m./ $100 \mu \mathrm{l}$ of the radioligand ${ }^{125} \mathrm{I}-\left[\mathrm{Tyr}^{10}\right]$ rat secretin $^{19}$ $(2000 \mathrm{Ci} / \mathrm{mmol}$; Anawa, Wangen, Switzerland). Nonspecific radioligand binding was assessed by incubating tissue sections in the incubation solution containing $100 \mathrm{nM}$ of non-radiolabeled (cold) human secretin in addition to ${ }^{125} \mathrm{I}-\left[\mathrm{Tyr}^{10}\right]$ rat secretin. At this concentration, cold secretin completely and specifically displaces ${ }^{125} \mathrm{I}-\left[\mathrm{Tyr}^{10}\right]$ rat secretin at the receptors. Further competition experiments were performed to distinguish secretin receptors from other receptors of the secretin receptor family, which bind secretin with low affinity. ${ }^{20}$ For this purpose, serial tissue sections were incubated with ${ }^{125} \mathrm{I}-\left[\mathrm{Tyr}^{10}\right]$ rat secretin and increasing concentrations of one of the following cold peptides: human secretin, VIP (Bachem, Bubendorf, Switzerland) or glucagon(1-29) (Bachem). After incubation, the slides were washed five times in ice-cold HEPES containing 1\% BSA and twice in ice-cold HEPES without BSA. The slides were dried for $15 \mathrm{~min}$ under a stream of cold air and then exposed to Kodak films Biomax $\mathrm{MR}^{\circledR}$ for 7 days at $4^{\circ} \mathrm{C}$. The signals on the films were analyzed in correlation with morphology, using a corresponding H\&Estained tissue section. The signal density was quantitatively assessed using tissue standards for iodinated compounds (Amersham, Aylesbury, UK), and using a computer-assisted image processing system (Analysis Imaging System, Interfocus, Mering, Germany). In all experiments, rat pancreas served as positive control. ${ }^{14}$

\section{RT-PCR Analysis of Secretin Receptor Transcripts}

In representative cases, the receptor autoradiography results were confirmed by RT-PCR specific for secretin receptor transcripts. These cases included three carcinoid tumors and three non-neoplastic lung samples with positive secretin receptor autoradiography, and three carcinoid tumors, three NSCLC, three SCLC, and three non-neoplastic lung samples with negative secretin receptor autoradiography. An area of either tumor tissue or non-neoplastic lung tissue was selected on an H\&E-stained tissue section and cut out of the frozen tissue block with a sterile scalpel blade. The dissected frozen samples were weighed followed by grinding in liquid nitrogen. The resulting tissue powders were decanted into microfuge tubes and resuspended in Trizol reagent (Invitrogen, Carlsbad, CA, USA) to a final concentration of $50 \mu \mathrm{g}$ tissue per $1 \mathrm{ml}$ Trizol. Total cellular RNA was isolated from 
$250 \mu \mathrm{l}$ of each Trizol suspension using the RNEasy Mini kit (Qiagen, Valencia, CA, USA). Purified RNA was digested with amplification-grade deoxyribonuclease I (DNAse I; Invitrogen) for $15 \mathrm{~min}$ at room temperature to remove any genomic DNA contamina tion. Subsequently, EDTA was added ( $2 \mathrm{mM}$ final; Invitrogen) and the samples were heated to $65^{\circ} \mathrm{C}$ for $15 \mathrm{~min}$ to inactivate the DNAse I. One-half microgram of each purified RNA was used to synthesize cDNA via the Reverse Transcription System (Promega, Madison, WI, USA) and approximately $25 \mathrm{ng}$ of resultant cDNA was used as template for the ensuing PCR reactions. Amplification of human secretin receptor transcripts was performed as previously described, ${ }^{9}$ using $5^{\prime}$ and $3^{\prime}$ primers corresponding to nucleotides 107-127 and 1434-1413, respectively, under GenBank accession number U28281(sense, $5^{\prime}$-CCATGCGTCCCCACCTGTCGC- $3^{\prime}$ and antisense, $5^{\prime}$-CTCTCAGATGATGCTGGTCCTG-3'). Control reactions were performed using previously described human secretin receptor isoforms (wild-type human secretin receptor and human secretin receptor$\Delta$ exon 3) cloned into the pBK-CMV vector, ${ }^{9}$ or with no template cDNA (water only). Actin PCR reactions were run using the following primer pair: sense, $5^{\prime}$-CCAGCTCACCATGGATGATGATATCG-3' ${ }^{\prime}$ and antisense, $5^{\prime}$-GGAGTTGAAGGTAGTTTCGTGGATGC- ${ }^{\prime}$. PCR reactions were run in $50-\mu \mathrm{l}$ volume containing $0.2 \mu \mathrm{M}$ of both sense and antisense primers, $2 \mathrm{mM}$ $\mathrm{MgCl}_{2}$ (Invitrogen), $0.2 \mathrm{mM}$ dNTPs (Stratagene, La Jolla, CA, USA), and 0.5 U of Platinum Taq Polymerase (Invitrogen). Reactions were performed in a DNA Engine (MJ Research, South San Francisco, CA, USA), using optimized cycle conditions comprised of an initial denaturing step of $2 \mathrm{~min}$ at $94^{\circ} \mathrm{C}$ and subsequently 32 cycles of $94^{\circ} \mathrm{C}$ for $30 \mathrm{~s}, 64^{\circ} \mathrm{C}$ for $30 \mathrm{~s}$, and $72^{\circ} \mathrm{C}$ for $2 \mathrm{~min}$. Fifteen-microliter fractions of each amplification reaction were resolved on a $2 \%$ agarose/TAE gel along with the $1 \mathrm{~kb}$ Plus ladder (Invitrogen) as marker. The identities of the prominent and reproducible PCR products were determined by ligating $1 \mu \mathrm{l}$ of relevant PCR reactions directly into PCR 2.1 TOPO cloning vector (Invitrogen), followed by bacterial transformation. DNA was then isolated, digested with EcoRI, and constructs having inserts with the relevant sizes were characterized by direct sequencing. Sequences were analyzed relevant to the well-established genomic and cDNA sequences for the secretin receptor, as well as broad BLAST analysis against the human genome.

\section{Results}

\section{Secretin Receptor Protein in Lung Tumors}

The secretin receptor protein expression in lung tumors was assessed on the basis of receptor binding, by in vitro receptor autoradiography. Table 1 and Figure 1 summarize the secretin receptor incidences and densities. The highest secretin
Table 1 Secretin receptor incidence and density in lung tumors and mesotheliomas

\begin{tabular}{|c|c|c|}
\hline & $\begin{array}{l}\text { Secretin } \\
\text { receptor } \\
\text { incidence } \\
\text { Positive/total } \\
\text { cases }(\%)\end{array}$ & $\begin{array}{c}\text { Secretin receptor density } \\
\text { Mean } \pm \text { s.e.m. (range) } \\
\text { of receptor positive } \\
\text { cases (d.p.m. per mg } \\
\text { tissue) }\end{array}$ \\
\hline \multicolumn{3}{|l|}{ Carcinoid tumors } \\
\hline $\begin{array}{l}\text { Typical carcinoid } \\
\text { tumors }\end{array}$ & $17 / 22(77 \%)$ & $1955 \pm 421(344-5816)$ \\
\hline $\begin{array}{l}\text { Atypical carcinoid } \\
\text { tumors }\end{array}$ & $1 / 7(14 \%)$ & 5252 \\
\hline Total & $18 / 29(62 \%)$ & $2138 \pm 437$ \\
\hline \multicolumn{3}{|l|}{ NSCLC } \\
\hline $\begin{array}{l}\text { Squamous cell } \\
\text { carcinomas }\end{array}$ & $1 / 15(7 \%)$ & 402 \\
\hline Adenocarcinomas & $2 / 11(18 \%)$ & $994 \pm 663(331-1656)$ \\
\hline Total & $3 / 26(12 \%)$ & $796 \pm 430$ \\
\hline$S C L C$ & $0 / 10(0 \%)$ & - \\
\hline \multicolumn{3}{|l|}{ Mesotheliomas } \\
\hline Pleural & $0 / 10(0 \%)$ & - \\
\hline Peritoneal & $1 / 1$ & 1351 \\
\hline
\end{tabular}

NSCLC, non-small cell lung cancer; SCLC, small cell lung cancer.

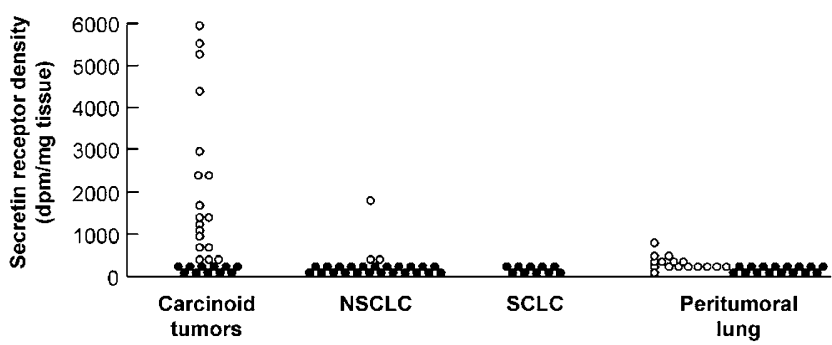

Figure 1 Secretin receptor density (d.p.m. per mg tissue) in bronchopulmonary carcinoid tumors $(n=29)$, NSCLC $(n=26)$, SCLC $(n=10)$, and peritumoral lung alveoli $(n=32)$ as measured by quantitative secretin receptor autoradiography. The white dots correspond to secretin receptor-positive cases, and the black dots to secretin receptor-negative cases. The secretin receptor density ranges from low to high in carcinoid tumors and from low to moderate in NSCLC, whereas it is consistently low in alveoli. All SCLC are receptor-negative.

receptor expression was found in bronchopulmonary carcinoid tumors, with sixty-two percent showing secretin receptors in often moderate or high density. The receptor incidence, but not density, was significantly lower in atypical carcinoids than in typical carcinoids. In contrast to the carcinoid tumors, NSCLC expressed secretin receptors in only rare cases and low density, and no SCLC was found to be receptor-positive. Regarding mesotheliomas, secretin receptors were not identified in any of the pleural tumors, but were identified in the single investigated peritoneal tumor. Figure 2 illustrates the autoradiography results with representative examples of various tumor types. In the carcinoid tumor in the top row, strong secretin binding is present in the entire tumor sample, whereas the 


\section{Bronchopulmonary carcinoid tumor}
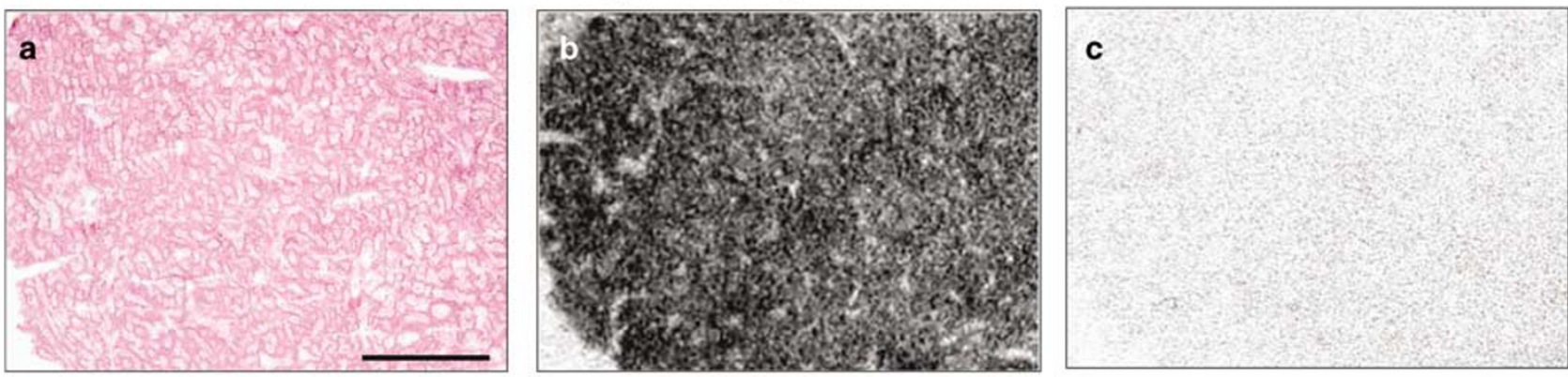

\section{Non-small cell lung cancer}
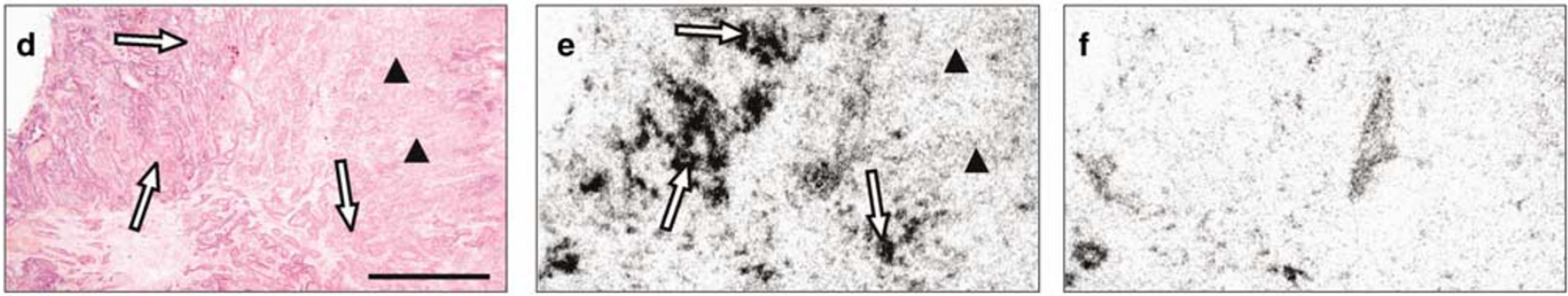

\section{Small cell lung cancer}
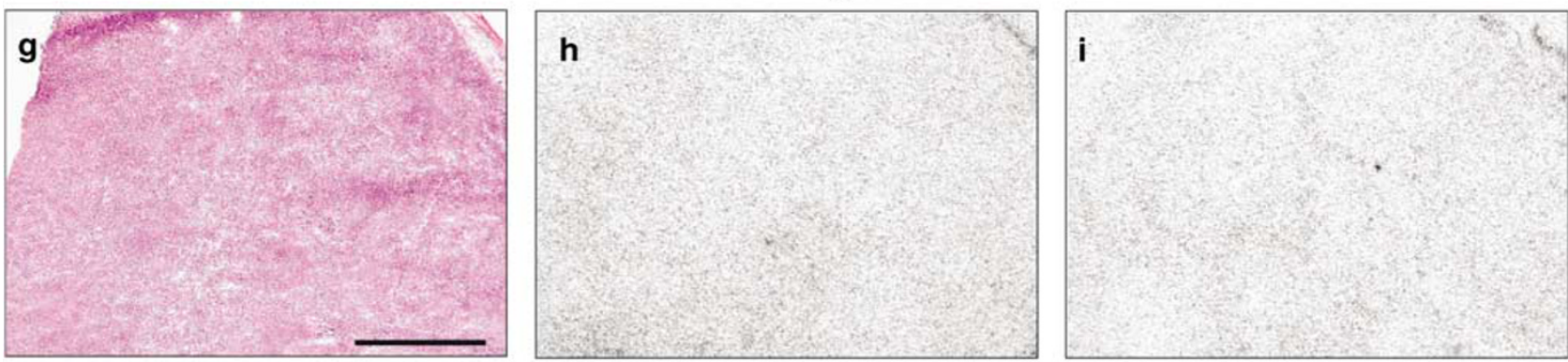

\section{Peritoneal mesothelioma}

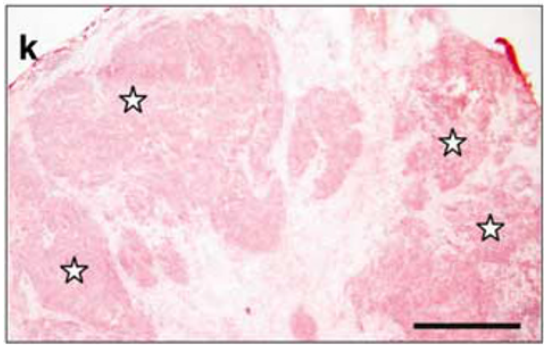

HE

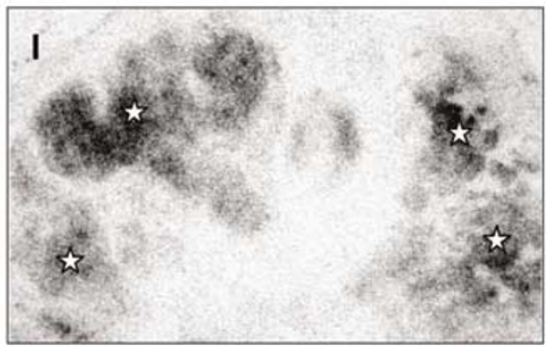

Total

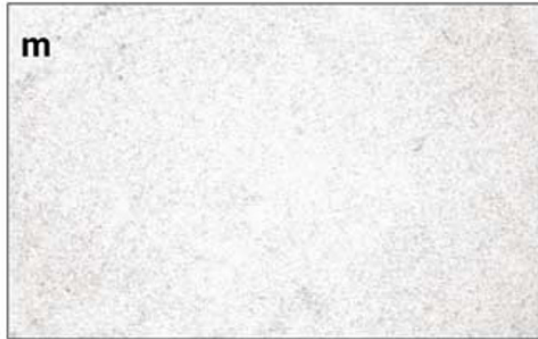

Non-specific

Figure 2 In vitro secretin receptor autoradiography on serial tissue sections to assess ${ }^{125} \mathrm{I}-\left[\mathrm{Tyr}^{10}\right]$ rat secretin binding to lung tumors, and a peritoneal mesothelioma. (a, d, g, k) H\&E-stained tissue sections showing the following: a bronchopulmonary typical carcinoid tumor (a), an NSCLC, namely an adenocarcinoma (d) (arrows and triangles), an SCLC (g), and a peritoneal mesothelioma (k) (asterisks). Bars $=1 \mathrm{~mm}$. (b, e, h, l) Autoradiograms showing total binding of ${ }^{125} \mathrm{I}$-[Tyr $\left.{ }^{10}\right]$ rat secretin to tumors. (b) Very strong and homogeneous binding to the carcinoid tumor; (e) heterogeneous binding with high (arrows) and low (triangles) receptor densities in the NSCLC; (h) no binding to the SCLC; (l) moderate to strong binding to the mesothelioma. (c, f, i, m) Autoradiograms showing non-specific binding of ${ }^{125} \mathrm{I}-\left[\mathrm{Tyr}^{10}\right]$ rat secretin in the presence of $100 \mathrm{nM}$ cold human secretin. Complete displacement of ${ }^{125} \mathrm{I}-\left[\mathrm{Tyr}^{10}\right]$ rat secretin by ${ }^{\mathrm{cold}}$ secretin provides proof of specific receptor binding in all four cases.

NSCLC in the second row shows substantial secretin binding in only a part of the tumor sample, and very weak secretin binding in the remaining tumor tissue. The SCLC in the third row displays no and the peritoneal mesothelioma in the bottom row moderate secretin binding. 


\section{Secretin Receptor Protein in the Non-Neoplastic Lung}

Secretin receptors were also found in the nonneoplastic lung by secretin receptor autoradiography. They were expressed along the alveolar septa but not in peripheral bronchi, arteries, veins, or pleura (Table 2). Of note, proximal bronchi that were previously shown to express secretin receptors, ${ }^{16}$ were not available in the investigated materials. Importantly, the secretin receptor expression along the alveolar septa was not found in the entire investigated lung parenchyma, but was restricted to specific lung areas. First, it was exclusively present in direct vicinity of tumors, that is, within a few centimeters distance from the tumors, but not farther away. Second, it was identified only in cases, which histologically showed a prominent intraalveolar neutrophilic exudate, but not in cases without acute inflammation. This is illustrated with typical examples in Figure 3: the peritumoral lung samples with intra-alveolar neutrophilic exudate showed a weak diffuse secretin receptor expression along the alveolar septa (Figure 3a-g), whereas the histologically normal lung sample obtained at a distance from a tumor was receptor-negative (Figure $3 \mathrm{~h}-\mathrm{l}$ ). The secretin receptor density in the peritumoral alveoli was low, often much lower than in the carcinoid tumors, as illustrated in Figure 1.

\section{Pharmacological Characterization of Secretin Receptors}

Secretin is specifically bound not only by secretin receptors with high affinity, but also by other receptors of the secretin receptor family, such as VIP and glucagon receptors, with low affinity. ${ }^{14}$ Therefore, it had to be proven that in the investigated tissues the radioligand ${ }^{125} \mathrm{I}-\left[\mathrm{Tyr}^{10}\right]$ rat secretin was bound solely by secretin receptors. For this purpose, pharmacological competition experiments were performed to assess the rank order of potencies of secretin, VIP, and glucagon at the identified receptors. In all secretin-binding tumors and

Table 2 Secretin receptor incidence and density in the nonneoplastic human lung

\begin{tabular}{lcc}
\hline & $\begin{array}{c}\text { Secretin receptor } \\
\text { incidence } \\
\text { Positive/total } \\
\text { samples (\%) }\end{array}$ & $\begin{array}{c}\text { Secretin receptor density } \\
\text { Mean } \pm \text { s.e.m. (range) of } \\
\text { receptor positive cases } \\
\text { (d.p.m. per mg tissue) }\end{array}$ \\
\hline $\begin{array}{l}\text { Alveoli } \\
\text { peritumoral }\end{array}$ & $16 / 32(50 \%)$ & $329 \pm 42(133-852)$ \\
$\begin{array}{l}\text { Alveoli remote } \\
\text { from tumor }\end{array}$ & $0 / 16(0 \%)$ & - \\
$\begin{array}{l}\text { Bronchioli } \\
\text { Bronchi }\end{array}$ & $0 / 32(0 \%)$ & - \\
$\begin{array}{l}\text { Pulmonary arteries } \\
\text { Pulmonary veins }\end{array}$ & $0 / 8(0 \%)$ & - \\
Pleura & $0 / 34(0 \%)$ & - \\
\hline
\end{tabular}

non-neoplastic lung tissues, ${ }^{125} \mathrm{I}-\left[\mathrm{Tyr}^{10}\right]$ rat secretin was displaced by cold secretin with high affinity in the nanomolar concentration range, and by VIP and glucagon with low affinity in the micromolar concentration range, as shown by two representative displacement curves in Figure 4. This rank order of potencies provides strong pharmacological evidence that secretin receptors were specifically identified. ${ }^{14}$

\section{Secretin Receptor Transcripts in Lung Tumors and Non-Neoplastic Lung}

To further confirm the autoradiography results and to assess the occurrence of secretin receptor mis-splicing in the investigated tissues, RT-PCR analysis of secretin receptor mRNAs was performed in selected cases (Figure 5). Carcinoid tumors chosen on the basis of strong secretin binding in the autoradiography experiments, as well as peritumoral non-neoplastic lung tissues with secretin binding, all showed transcripts for the wild-type secretin receptor. The bands were very strong in carcinoid tumors and less intense in peritumoral lung tissues. In addition, various secretin receptor splice variants, as documented by sequence analysis, were found in these tissues. In carcinoid tumors, the splice variants included one with deletion of exon 9 and one with tandem deletion of exons 2 and 3 . In peritumoral lung, a different spliceoform was identified as missing part of exon 1 and all of exons 2-4. All these secretin receptor spliceoforms have not previously been reported or characterized. The exon 9 deletion would be expected to yield early truncation of the mature protein after the third transmembrane segment, within the second extracellular loop, just before the conserved cysteine residue that is typically within that loop and involved in a functionally important disulfide bond. Due to a shift in the reading frame, the resulting protein is truncated just six residues later. This receptor variant would likely not signal in response to secretin. Both the $\Delta$ exon2,3 and the $\Delta$ exon2,3,4 splice variants would be expected to remain in frame, but would also not bind secretin or signal in response to secretin. Interestingly, secretin receptor splice-variant transcripts previously identified in pancreatic and liver cancers, such as transcripts with exon 3 deletion and exon 3 and 4 deletion, ${ }^{10-12}$ were not detected in any lung tissues.

Tissues without secretin binding by autoradiography, including carcinoid tumors, NSCLC, SCLC, and non-neoplastic lung, expressed no secretin receptor mRNA either for the wild-type receptor or for receptor splice variants.

\section{Discussion}

In the present study, the secretin receptor, a gastrointestinal peptide hormone receptor and 


\section{Peritumoral lung}
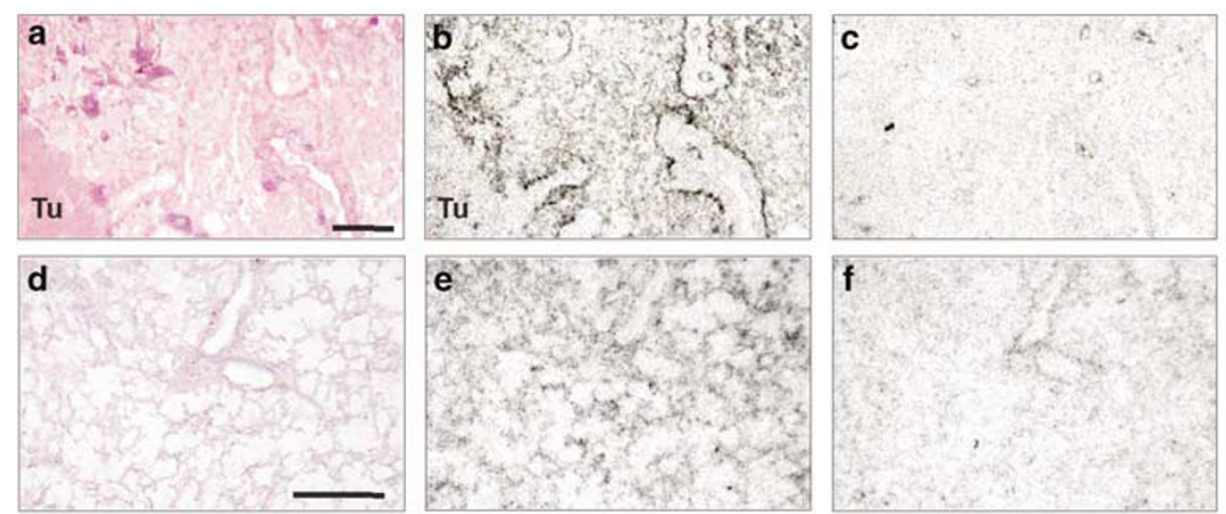

\section{Lung remote from tumor}

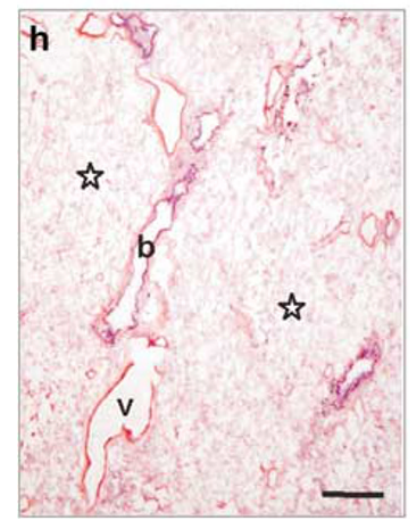

HE

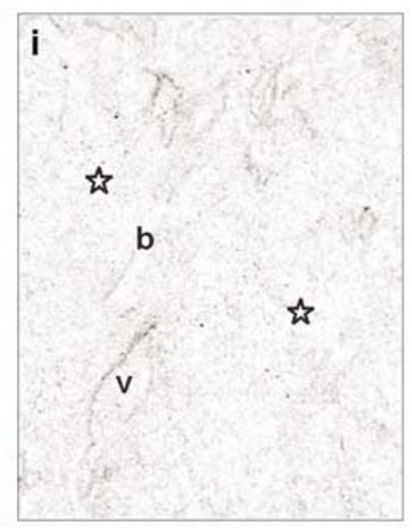

Total

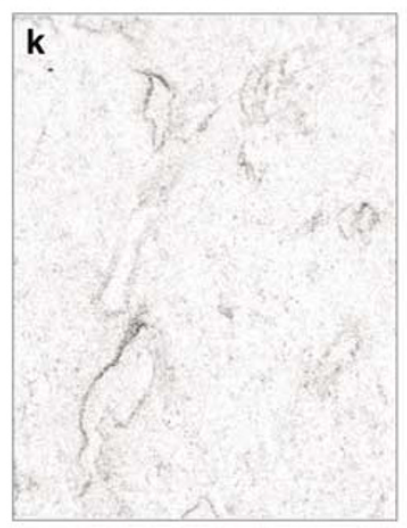

Non-specific
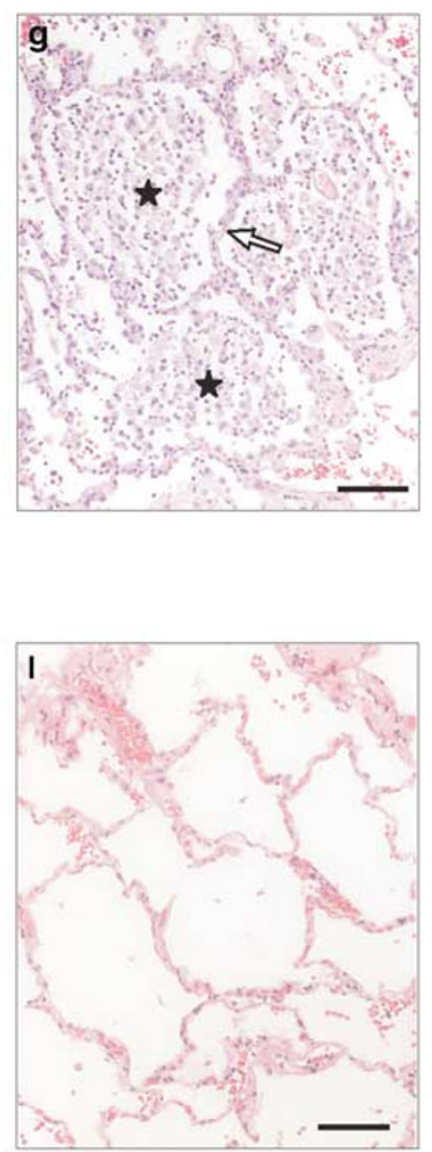

HE

Figure 3 (a-c, $\mathbf{d}-\mathbf{f}, \mathbf{h}-\mathbf{k})$ In vitro secretin receptor autoradiography on serial tissue sections of non-neoplastic human lung tissues. (a, d, h) H\&E-stained tissue sections showing the following: lung parenchyma with alveoli (asterisks) and intralobular septa (arrows) adjacent to an NSCLC (Tu) (a), peritumoral alveoli (asterisks) and blood vessels (v) (d), and lung parenchyma remote from tumor with alveoli (asterisks), bronchi (b), and blood vessels (v) (h). Bars $=1 \mathrm{~mm}$. (b, e, i) Autoradiograms showing total binding of ${ }^{125} \mathrm{I}-\left[\mathrm{Tyr}{ }^{10}\right]$ rat secretin. (b) In the peritumoral lung, diffuse very weak binding to the alveolar area (asterisks), and more pronounced binding to the outer contours of intralobular septa (arrows); no binding to tumor. (e) Higher magnification of peritumoral lung reveals that $\left.{ }^{125} \mathrm{I}_{-[\mathrm{Tyr}}{ }^{10}\right]$ rat secretin binds along alveolar outlines (asterisks); no binding to blood vessels. (i) Remote from tumor, no binding to the alveoli, bronchi, or blood vessels. (c, f, k) Autoradiograms showing non-specific ${ }^{125} \mathrm{I}_{-}\left[\mathrm{Tyr}^{10}\right]$ rat secretin binding in the presence of $100 \mathrm{nM}$ cold secretin. (g, l) H\&E-stained tissue sections of the same cases at high magnification. Bars =0.1 mm. (d) Secretin receptor-positive peritumoral lung exhibits prominent neutrophilic exudate in alveolar lumina (asterisks; arrow indicates alveolar septum), whereas (h) lung alveoli without secretin binding show normal histology.

a recently recognized marker for pancreatic and biliary tract cancer, ${ }^{8-12}$ is for the first time systematically assessed in the lung. The study yields three main results. In lung neoplasia, secretin receptors are highly expressed in carcinoid tumors, but scarcely or not in NSCLC, SCLC, or mesotheliomas. In the non-neoplastic lung, secretin receptors are observed in the alveolar septa under pathological conditions, namely in peritumoral acute inflammation. Finally, specific secretin receptor mis-splicing occurs in tumor and non-tumor lung pathology, but not in the normal lung. Given that the lung is embryologically related to the foregut, it is not completely surprising to find a classical gut hormone receptor to be expressed also in the lung.
In the gastrointestinal tract, however, secretin receptors show a remarkably higher physiological expression than in the lung. ${ }^{10,11,16}$ Moreover, the secretin receptor spliceoforms identified in the lung differ from those found in the pancreas and liver. ${ }^{8-12}$

Proof of specific secretin receptor identification is provided at both the protein and mRNA levels. In the in vitro receptor autoradiography experiments assessing secretin receptor protein on the basis of secretin binding, it is important to demonstrate that secretin is not bound by VIP receptors, which have a low affinity for secretin and are known to be expressed in NSCLC and the normal human lung. ${ }^{20,21}$ Therefore, we performed pharmacological competition experiments, which show a rank 

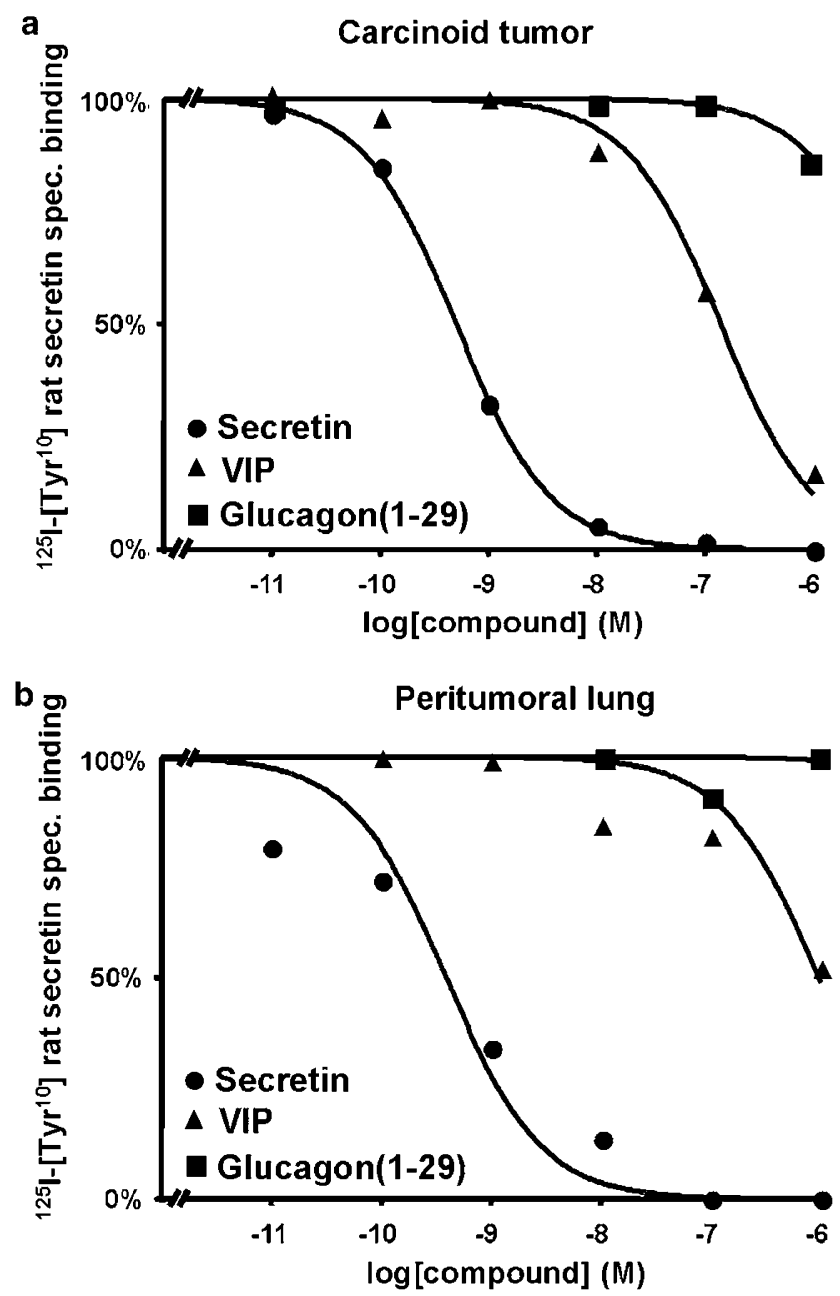

Figure 4 Representative pharmacological competition experiments showing displacement of ${ }^{125} \mathrm{I}_{-}\left[\mathrm{Tyr}^{10}\right]$ rat secretin by cold secretin, VIP, and glucagon(1-29) in (a) a bronchopulmonary carcinoid tumor and (b) peritumoral non-neoplastic alveoli. Highaffinity displacement of ${ }^{125} \mathrm{I}-\left[\mathrm{Tyr}^{10}\right]$ rat secretin by human secretin (๑), but low-affinity displacement by VIP $(\boldsymbol{\Lambda})$ and glucagon(1-29) $(\boldsymbol{\square})$, provides evidence of specific secretin receptor binding.

order of potencies (secretin $\gg$ VIP $>$ glucagon) characteristic of secretin receptors. Moreover, tissues with known VIP receptor expression, such as NSCLC and normal lung, ${ }^{21}$ are negative for secretin receptors (data not shown). This provides strong evidence that the receptors identified in the current study are secretin receptors. ${ }^{14,22}$ In addition, RT-PCR fully confirms the autoradiography results. Secretin receptor mRNA is identified in tissues with, but not in tissues without, secretin receptor binding.

Secretin receptors are new markers for carcinoid tumors of the lungs. Biologically, secretin receptors may mediate functional effects of secretin on carcinoid tumor cells. These effects may include for instance hormone secretion, first, in analogy to gastrinomas, ${ }^{8,23}$ and second, because several hormones produced by lung carcinoid tumors are physiologically regulated by secretin. ${ }^{24-26}$ Furthermore, secretin receptor activation, previously shown to inhibit cell proliferation in a transfected cell line, ${ }^{9}$ may also be linked to proliferation of lung carcinoid tumors. Another important aspect of the presence of secretin receptors in lung carcinoid tumors is the potential for clinical applications, namely for an in vivo secretin receptor targeting of lung carcinoid tumors analogous to the somatostatin receptor targeting of gut endocrine tumors. ${ }^{1}$ A targeted scintigraphy with adequately radiolabeled secretin analogs may be used for imaging. This may be helpful for staging and follow-up of carcinoid tumors, as well as for the differential diagnosis between carcinoid tumors and NSCLC and SCLC. ${ }^{27}$ Advantageous for a secretin receptor-targeted scintigraphy of lung tumors would be the very low secretin receptor expression in the peritumoral lung, which could be expected to yield only very little background signals in vivo. A further extension of this principle may be a targeted radiotherapy with radiotoxic secretin analogs as new treatment option for lung carcinoid tumors, which can have an unfavorable outcome and only poorly respond to chemotherapy. ${ }^{27,28}$

Bronchopulmonary carcinoid tumors not only express the wild-type secretin receptor, but also several secretin receptor splice variants. Mis-splicing of the secretin receptor pre-mRNA was observed previously in pancreatic and cholangiocellular cancer. ${ }^{9-12}$ In the present study, however, none of the known secretin receptor spliceoforms, but two novel splice variants, which have not been found in other tumors before, were identified in lung carcinoid tumors, including a variant with exon 9 deletion and a variant with exon 2 and 3 deletion. Thus, secretin receptor mis-splicing could be organ- and tumor-specific. It may have the potential to be used for tumor diagnosis and therapy, based on the preliminary data on gut cancer: the $\Delta$ exon3,4 secretin receptor spliceoform has recently been identified as serum biomarker for tumors, ${ }^{12}$ and the $\Delta$ exon3 variant, non-functional by itself, may affect specific biological characteristics of the tumor cells by silencing the wild-type receptor. ${ }^{8,9}$ As for lung carcinoid tumors, the two novel splice variants that would be expected not to bind secretin or to signal in response to secretin, need to be more thoroughly characterized in follow-up studies. It should, however, be emphasized that in lung carcinoid tumors (and in peritumoral lung) the wild-type secretin receptor remains the much predominant secretin receptor type compared with splice variants.

This study reports for the first time a secretin receptor expression in the alveolar septa in the peritumoral lung. Although it is difficult to precisely identify the cell type in the alveolar wall expressing secretin receptors, due to resolution limitations of receptor autoradiography, we can exclude a secretin receptor expression in contaminating tumor cells, carcinoid tumorlets, or neuroendocrine cell hyperplasia by morphology and based 


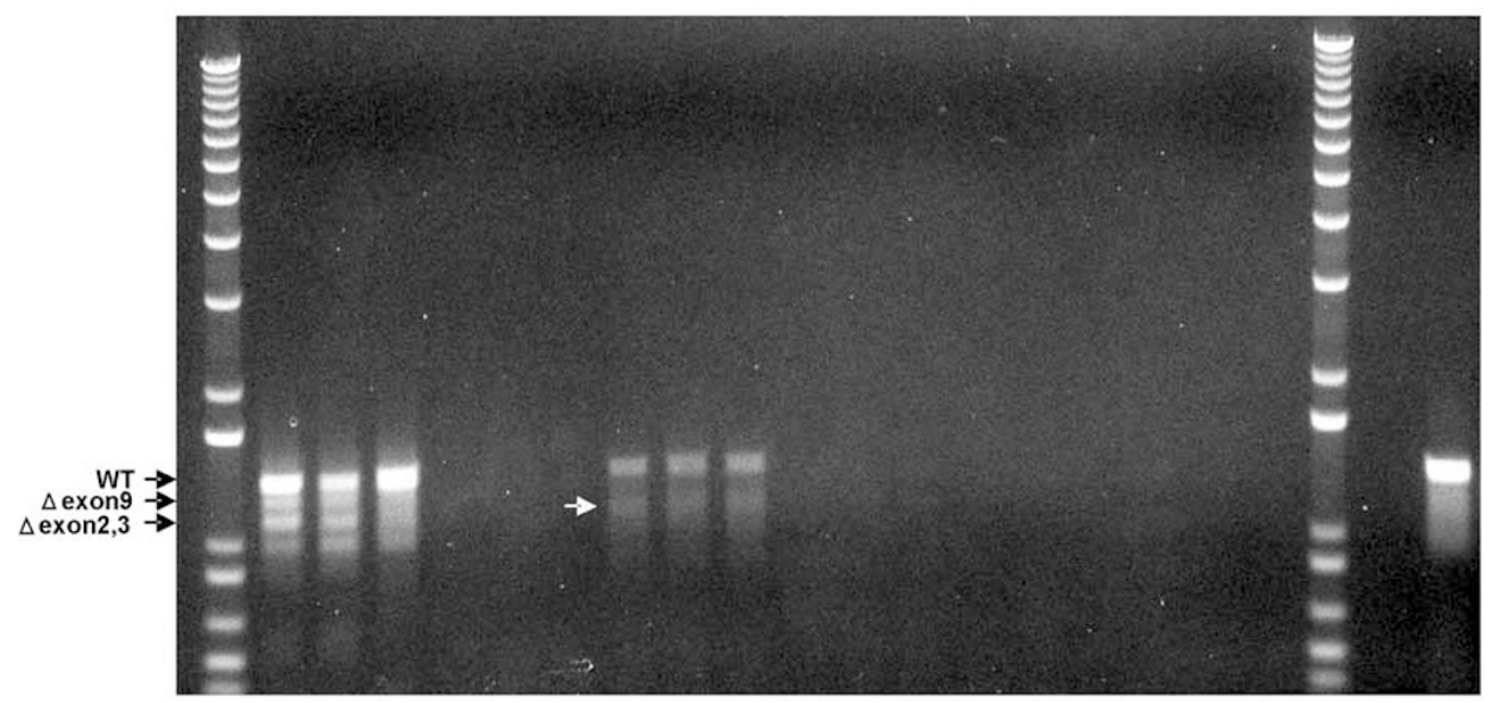

b

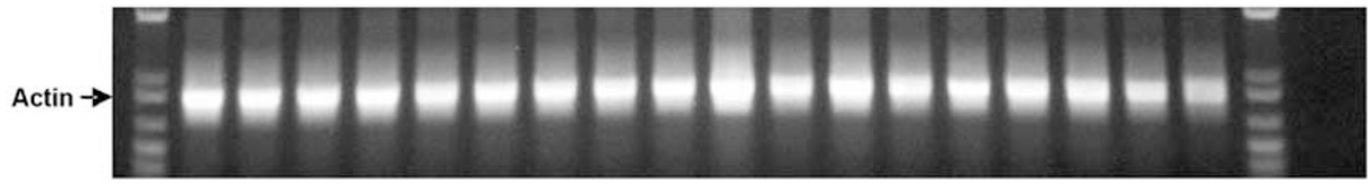

Figure 5 RT-PCR for secretin receptor transcripts in bronchopulmonary carcinoid tumors with high-density secretin receptor binding $(\mathrm{ARG}+)$ and without secretin receptor binding (ARG-), in non-neoplastic lung with low-density secretin receptor binding (ARG +) and without secretin receptor binding (ARG-), and in SCLC and NSCLC without secretin receptor binding. RT-PCR was performed using primers able to amplify (a) full-length secretin receptor transcripts or (b) actin. Control reactions were run utilizing wild-type secretin receptor cloned into the pBK-CMV expression plasmid or with no template added (negative control). The 1-kb Plus ladder from Invitrogen was included as a molecular weight marker. The autoradiographically positive carcinoid tumors showed a strong band corresponding to wild-type secretin receptor transcripts, as well as weaker bands corresponding to secretin receptor splice variants with deletion of exon 9 and deletion of exons 2 and 3 in tandem, respectively. Both variants were proven by direct sequencing. In the autoradiographically positive peritumoral lung tissues, there was a stronger band representing wild-type receptor transcripts and a weaker band corresponding to a secretin receptor spliceoform missing part of exon 1 as well as exons 2-4 (white arrow). Conversely, no secretin receptor transcripts, either for the wild-type receptor or for spliceoforms, were identified in the autoradiographically negative carcinoid tumors, non-neoplastic lung, SCLC, and NSCLC.

on the gross tissue distribution patterns of neuroendocrine cell lesion and secretin receptors. ${ }^{29}$ Based on close molecular and functional similarities between pneumocytes and secretin receptor expressing cholangiocytes, ${ }^{30-32}$ we may propose that putative secretin receptor-expressing alveolar epithelial cells regulate transcellular water transport. Furthermore, evidence of secretin receptor mis-splicing was found in these pathological lung tissues. The detected spliceoform with deletion of part of exon 1 and of exons 2-4 is different from that detected in chronic pancreatitis, ${ }^{12}$ and has uptil now not been identified in other organs or tumors.

In summary, secretin receptors are increasingly being recognized as specifically expressed in human tumor and non-tumor pathologies of various organs. This represents the molecular basis for potential clinical applications. High tumoral secretin receptor binding may be used for an in vivo targeting of tumors with radiolabeled secretin analogs for diagnostic and therapeutic purposes. Further studies are 
needed to advance our understanding of the role of secretin receptor mRNA mis-splicing in pathology, and to help decide how we might begin to take advantage of this, clinically.

\section{Acknowledgements}

We thank Renee Happs and Beatrice Waser for technical assistance. This study was partially supported by the National Institutes of Health Grant DK46577.

\section{References}

1 Reubi JC. Peptide receptors as molecular targets for cancer diagnosis and therapy. Endocr Rev 2003;24: 389-427.

2 Krenning EP, Kwekkeboom DJ, Pauwels S, et al. Somatostatin receptor scintigraphy. In: Freeman LM (ed). Nuclear Medicine Annual, 1995. Raven Press: New York, 1995, pp 1-50.

3 Gibril F, Reynolds JC, Doppman JL, et al. Somatostatin receptor scintigraphy: its sensitivity compared with that of other imaging methods in detecting primary and metastatic gastrinomas. Ann Intern Med 1996; 125:26-34.

4 Waldherr C, Pless M, Maecke HR, et al. Tumor response and clinical benefit in neuroendocrine tumors after 7.4 GBq (90)Y-DOTATOC. J Nucl Med 2002;43:610-616.

5 Kwekkeboom DJ, Teunissen JJ, Bakker WH, et al. Radiolabeled somatostatin analog [177Lu-DOTA0, Tyr3] octreotate in patients with endocrine gastroenteropancreatic tumors. J Clin Oncol 2005;23:2754-2762.

6 Harmar AJ. Family-B G-protein-coupled receptors. Genome Biol 2001;2:REVIEWS3013.1-REVIEWS3013.10.

7 Chey WY, Chang TM. Secretin, 100 years later. J Gastroenterol 2003;38:1025-1035.

8 Ding WQ, Kuntz S, Bohmig M, et al. Dominant negative action of an abnormal secretin receptor arising from mRNA missplicing in a gastrinoma. Gastroenterology 2002;122:500-511.

9 Ding WQ, Cheng ZJ, McElhiney J, et al. Silencing of secretin receptor function by dimerization with a misspliced variant secretin receptor in ductal pancreatic adenocarcinoma. Cancer Res 2002;62:5223-5229.

10 Körner M, Hayes GM, Rehmann R, et al. Secretin receptors in normal and diseased human pancreas: marked reduction of receptor binding in ductal neoplasia. Am J Pathol 2005;167:959-968.

11 Körner M, Hayes GM, Rehmann R, et al. Secretin receptors in the human liver: expression in biliary tract and cholangiocarcinoma, but not in hepatocytes or hepatocellular carcinoma. J Hepatol 2006;45:825-835.

12 Hayes GM, Carrigan PE, Dong M, et al. A novel secretin receptor splice variant potentially useful for early diagnosis of pancreatic carcinoma. Gastroenterology 2007;133:853-861.

13 Chiba T, Yamatani T, Yamaguchi A, et al. Mechanism for increase of gastrin release by secretin in Zollinger-Ellison syndrome. Gastroenterology 1989; 96:1439-1444.
14 Patel DR, Kong Y, Sreedharan SP. Molecular cloning and expression of a human secretin receptor. Mol Pharmacol 1995;47:467-473.

15 Chow BK. Molecular cloning and functional characterization of a human secretin receptor. Biochem Biophys Res Commun 1995;212:204-211.

16 Davis RJ, Page KJ, Dos Santos Cruz GJ, et al. Expression and functions of the duodenal peptide secretin and its receptor in human lung. Am J Respir Cell Mol Biol 2004;31:302-308.

17 Beasley MB, Thunnissen FB, Hasleton $\mathrm{P}$, et al. Carcinoid tumour. In: Travis WD, Brambilla E, Müller-Hermelink HK, Harris CC (eds). Pathology and Genetics of Tumours of the Lung, Pleura, Thymus and Heart. IARC Press: Lyon, 2004, pp 59-62.

18 Klöppel G. Tumour biology and histopathology of neuroendocrine tumours. Best Pract Res Clin Endocrinol Metab 2007;21:15-31.

19 Ulrich II CD, Wood P, Hadac EM, et al. Cellular distribution of secretin receptor expression in rat pancreas. Am J Physiol 1998;275:G1437-G1444.

20 Ulrich II CD, Holtmann M, Miller LJ. Secretin and vasoactive intestinal peptide receptors: members of a unique family of $\mathrm{G}$ protein-coupled receptors. Gastroenterology 1998;114:382-397.

21 Reubi JC, Läderach U, Waser B, et al. Vasoactive intestinal peptide/pituitary adenylate cyclase-activating peptide receptor subtypes in human tumors and their tissues of origin. Cancer Res 2000;60: 3105-3112.

22 Jiang S, Ulrich C. Molecular cloning and functional expression of a human pancreatic secretin receptor. Biochem Biophys Res Commun 1995;207:883-890.

23 Frucht H, Howard JM, Slaff JI, et al. Secretin and calcium provocative tests in the Zollinger-Ellison syndrome. A prospective study. Ann Intern Med 1989;111:713-722.

24 al-Saffar N, White A, Moore M, et al. Immunoreactivity of various peptides in typical and atypical bronchopulmonary carcinoid tumours. Br J Cancer 1988;58: $762-766$.

25 Hattori Y, Imamura M, Tobe T. Gastrin release from antral $\mathrm{G}$ cells stimulated with secretin. Am J Gastroenterol 1992;87:195-200.

26 Nussdorfer GG, Bahcelioglu M, Neri G, et al. Secretin, glucagon, gastric inhibitory polypeptide, parathyroid hormone, and related peptides in the regulation of the hypothalamus-pituitary-adrenal axis. Peptides 2000; 21:309-324.

27 McMullan DM, Wood DE. Pulmonary carcinoid tumors. Semin Thorac Cardiovasc Surg 2003;15: 289-300.

28 Beasley MB, Thunnissen FB, Brambilla E, et al. Pulmonary atypical carcinoid: predictors of survival in 106 cases. Hum Pathol 2000;31:1255-1265.

29 Sheard JDH, Gosney JR. Endocrine cells in tumourbearing lungs. Thorax 1996;51:721-726.

30 Kanno N, LeSage G, Glaser S, et al. Regulation of cholangiocyte bicarbonate secretion. Am J Physiol Gastrointest Liver Physiol 2001;281:G612-G625.

31 Matthay MA, Robriquet L, Fang X. Alveolar epithelium: role in lung fluid balance and acute lung injury. Proc Am Thorac Soc 2005;2:206-213.

32 O'Grady SM, Lee SY. Chloride and potassium channel function in alveolar epithelial cells. Am J Physiol Lung Cell Mol Physiol 2003;284:L689-L700. 